\title{
Maintenance Decision for the Key Parts of Machine Tool Using the Monitoring Information
}

\author{
Shuang Zhou*, Tao Zan, Min Wang and Congcong Guo \\ Beijing Key Laboratory of Advanced Manufacturing Technology, College of Mechanical Engineering and Applied \\ Electronics Technology, Beijing University of Technology, Beijing 100124, China \\ *Corresponding author
}

\begin{abstract}
Considering the monitoring information of the operation process of the machine tools, a maintenance decision model is established in this paper. Firstly, the monitoring information of the key parts of the machine tools is obtained by using data acquisition system. Based on the kurtosis value which are extracted from the monitoring information, the weibull proportional hazard model (WPHM) are proposed. In the model, the weibull distribution is regarded as the failure rate function. Then, the maintenance decision model can be obtained based on WPHM. Finally, according to the failure threshold in the decision model, the maintenance decision can be conducted. The results show that this maintenance decision model can not only improve the availability of the key parts greatly, but also make full use of the effective life of machine tools.
\end{abstract}

Keywords-monitoring information; kurtosis value; weibull proportional hazards models; maintenance decision

\section{INTRODUCTION}

The overall performance of the machine tools has been the most concern in the field of manufacturing. People not only pay attention to the high performance of the machine tools, but also look forward to the reliability of the key parts. Due to the inherent characteristics of machine tools, the reliability assessment of its key parts still has some issues need to be further in-depth. On the one hand, the composition of machine tool is very complicated, and it is difficult to realize the reliability deterioration law of machine tool by using direct theory. On the other hand, machine tools are typical of high reliability and long life products, the MTBF (mean time between failures) is higher, so the reliability of the machine tool is lack of effective means by the general environmental stress test [1]. The popular method is to put the life of components as a random variable, and then select an appropriate mathematical model, calculate the distribution function and probability density function, both the reliability and life function of the equipment are finally obtained. But it can only resolve reliability issues at the manufacturing stage, and when the machining is a dynamic process, which running states has a great influence on the service life of components [2].

Condition-based maintenance (CBM) is a new maintenance decision developed in recent years, many scholars has done a lot of work in the mathematical modeling methods of CBM, Such as random coefficient regression model, hidden Markov model, logistic regression model. Jaouher Ben Ali et al. [3] established a new approach based on Weibull distribution and artificial neural network which is validated by an application on bearings. C.C. Lin and H.Y. Tseng [4] applied basic monitoring techniques in traditional reliability estimation model, using CMAC neural network on a whole system to estimate the reliability and the health of the equipment. Biao $\mathrm{Lu}$ et al. [5] considered an integrated reliability model is built for the machine based on the proportional hazard model considering the effects of the degradation states of quality-related components on machine reliability.

Maintenance decision model based on weibull proportional hazard model (WPHM) emphasizes that machine maintenance strategy should considering to the real-time operational status. Firstly, the parameters in the model are estimated by using the monitoring data, and then the maintenance decision model of the maximum availability of the key parts is established. Finally, the decision-making process of online monitoring were given. Moreover, CBM method is come from the perspective of the individual to reflect the real-time system reliability, which better ensure the accuracy of maintenance decisions.

\section{Weibull Proportional Hazards Model And PARAMETER ESTIMATION}

\section{A. Weibull Proportional Hazards Model}

Proportional hazards model (PHM) was first proposed by Cox, as PHM can establish a good relationship with the process parameters of system and the system itself, so it is applied to the reliability analysis. The basic form of PHM [6] can be represented as follows:

$$
h(t, Z)=\lambda_{0}(\mathrm{t}) \exp \left(\gamma Z_{\mathrm{t}}\right)
$$

where $h(t, Z)$ is the hazard value, $\lambda 0(t)$ is the reference failure rate function, it has nothing to do with the environment covariates, $\mathrm{Z} t$ is the state characteristic quantity at time $\mathrm{t}$, and $\gamma$ is the regression parameter, it shows the influence of characteristic quantities on the system hazard

The proportional hazards model assumes that the characteristic quantity has multiplicative effect on the hazard function, then $\gamma \mathrm{Zt}$ is a linear combination of the characteristic quantities. It combines various state information when system is running, which can effectively obtain the reliability of the system and carry out the maintenance decision. 
Mechanical systems are not failure as abrupt as electronic products, which generally appear to decline with the increase of running time, and then slowly there is an increasing trend. The above process can be described by the Poisson distribution, exponential distribution, weibull distribution etc. By curve fitting shows that three shapes of failure rate function of weibull distribution can consistent failure rate bathtub curves, and it is flexible, with good elasticity, therefore, weibull distribution were used to description failure. The two parameter Weibull distribution can be written as follows:

$$
h(t)=\frac{\beta}{\eta}\left[\frac{t}{\eta}\right]^{\beta-1}
$$

where $\beta$ is the shape parameter, when $\beta>1$, the failure rate of the component increases with time, $\eta$ is the scale parameter, which relating to the size of the system load, the larger the load, the smaller its value, and vice versa.

The weibull distribution for failure rate function instead of $\lambda_{0}(t),(1)$ will be updated as follows:

$$
h(t, Z)=\frac{\beta}{\eta}\left[\frac{t}{\eta}\right]^{\beta-1} \exp \left(\gamma Z_{\mathrm{t}}\right)
$$

This is the WPHM, while the reliability function based on the state characteristic and time can be calculated using the following formula:

$$
\begin{aligned}
R(t, Z) & =\exp \left(-\int_{0}^{t} h\left(s, Z_{\mathrm{s}}\right) d s\right) \\
& =\exp \left(-\int_{0}^{t} \exp \left(\gamma Z_{t}\right) d\left(\frac{t}{\eta}\right)^{\beta}\right)
\end{aligned}
$$

\section{B. Parameter Estimation}

For the WPHM model, the key problem is how to solve the three unknown parameters $\beta, \eta$ and $\gamma$. As the maximum likelihood estimation method has obvious advantages in dealing with incomplete sample, so the maximum likelihood estimation method is used to estimate the parameters of WPHM.

Firstly, the likelihood function is constructed by the failure density function, which can be written as follows:

$$
f(t, Z)=h(t, Z) R(t, Z)
$$

So the likelihood function will be updated as follows:

$$
\begin{aligned}
L(\beta, \eta, \gamma)= & \prod_{i=1}^{r} h\left(t_{\mathrm{i}}, Z\left(t_{i}\right)\right) \prod_{j=1}^{N} R\left(t_{j}, Z\left(t_{j}\right)\right)=\prod_{i=1}^{r} \frac{\beta}{\eta}\left(\frac{\mathrm{t}_{i}}{\eta}\right)^{\beta-1} \\
& \exp \left(\gamma Z\left(t_{j}\right)\right) \prod_{j=1}^{N} \exp \left(-\left(\frac{t_{j}}{\eta}\right)^{\beta} \exp \left(\gamma Z\left(t_{j}\right)\right)\right)
\end{aligned}
$$

where $N$ is the total sample number, $r$ is represents the number of invalid data in the total, the remaining $N-r$ after the data truncated, which meaning the system in monitoring point did not happen failure phenomenon. While $Z\left(t_{i}\right)$ is the state characteristics quantity at $t_{i}$ moments in the failure data, $Z\left(t_{j}\right)$ is the state characteristics quantity at $t_{j}$ moments in the total.

In order to obtain the estimated value of three parameters $\hat{\beta}, \hat{\eta}$ and $\hat{\gamma}$, taking logarithm on both side for (6) and updated as follows:

$$
\begin{aligned}
\ln L & =r \ln \frac{\beta}{\eta}+\sum_{i=1}^{r} \ln \left(\frac{t_{i}}{\eta}\right)^{\beta-1}+\sum_{i=1}^{r} \gamma Z\left(t_{i}\right) \\
& -\sum_{j=1}^{N}\left[\left(\frac{t_{j}}{\eta}\right)^{\beta} \exp \left(\gamma Z\left(t_{j}\right)\right)\right]
\end{aligned}
$$

Then, since the likelihood function is increasing, so it gets the maximum value at the same value for $\hat{\beta}, \hat{\eta}$ and $\hat{\gamma}$. Newton-Raphson iteration method is used to estimate the parameters.

In the first step, do the partial derivative of (7) respectively for $\beta, \eta$ and $\gamma$, specific steps as follows:

$$
\begin{gathered}
\frac{\partial \ln L}{\partial \beta}=\frac{r}{\beta}+\sum_{i=1}^{r} \ln \left(\frac{t_{i}}{\eta}\right) \\
-\sum_{j=1}^{N}\left[\left(\frac{t_{j}}{\eta}\right)^{\beta} \ln \left(\frac{t_{j}}{\eta}\right) \exp \left(\gamma Z\left(t_{j}\right)\right)\right] \\
\frac{\partial \ln L}{\partial \eta}=-\frac{r \beta}{\beta}+\sum_{j=1}^{N}\left[\frac{\beta}{\eta}\left(\frac{t_{j}}{\eta}\right)^{\beta} \exp \left(\gamma Z\left(t_{j}\right)\right)\right] \\
\frac{\partial \ln L}{\partial \gamma}=\sum_{i=1}^{r} Z\left(t_{i}\right) \\
-\sum_{j=1}^{N}\left[Z\left(t_{j}\right)\left(\frac{t_{j}}{\eta}\right)^{\beta} \exp \left(\gamma Z\left(t_{j}\right)\right)\right]
\end{gathered}
$$
follows:

Get a first-order derivative matrix, it can be represented as

$$
g=\left(\begin{array}{c}
\frac{\partial \ln L}{\partial \beta} \\
\frac{\partial \ln L}{\partial \eta} \\
\frac{\partial \ln L}{\partial \gamma}
\end{array}\right)
$$

In the second step, do the second partial derivative of (8), (9) and (10) respectively for $\beta, \eta$ and $\gamma$, specific steps as follows: 


$$
\begin{aligned}
& \frac{\partial^{2} \ln L}{\partial \beta^{2}}=-\frac{r}{\beta^{2}}-\sum_{j=1}^{N}\left[\left(\frac{t_{j}}{\eta}\right)^{\beta}\left[\ln \left(\frac{t_{j}}{\eta}\right)\right]^{2} \exp \left(\gamma Z\left(t_{j}\right)\right)\right] \\
& \frac{\partial^{2} \ln L}{\partial \eta^{2}}=\frac{r \beta}{\eta^{2}}-\sum_{j=1}^{N}\left[\left(\frac{t_{j}}{\eta}\right)^{\beta} \frac{\beta^{2}+\beta}{\eta^{2}} \exp \left(\gamma Z\left(t_{j}\right)\right)\right] \\
& \frac{\partial^{2} \ln L}{\partial \beta \partial \eta}=-\frac{r}{\eta}+\sum_{j=1}^{N}\left[\frac{1}{\eta}\left(\frac{t_{j}}{\eta}\right)^{\beta}\left(1+\beta \ln t_{j}\right) \exp \left(\gamma Z\left(t_{j}\right)\right)\right] \\
& \frac{\partial^{2} \ln L}{\partial \beta \partial \gamma}=-\sum_{j=1}^{N}\left[\left(\frac{t_{j}}{\eta}\right)^{\beta} Z\left(t_{j}\right) \ln \left(\frac{t_{j}}{\eta}\right) \exp \left(\gamma Z\left(t_{j}\right)\right)\right] \\
& \frac{\partial^{2} \ln L}{\partial \eta \partial \beta}=-\frac{r}{\eta} \\
& +\sum_{j=1}^{N}\left[\frac{1}{\eta}\left(\frac{t_{j}}{\eta}\right)^{\beta}\left(1+\beta \ln \left(\frac{t_{j}}{\eta}\right)\right) \exp \left(\gamma Z\left(t_{j}\right)\right)\right] \\
& \frac{\partial^{2} \ln L}{\partial \eta \partial \gamma}=\sum_{j=1}^{N}\left[\left(\frac{\beta}{\eta}\right)\left(\frac{t_{j}}{\eta}\right)^{\beta} Z\left(t_{j}\right) \exp \left(\gamma Z\left(t_{j}\right)\right)\right] \\
& \frac{\partial^{2} \ln L}{\partial \gamma \partial \beta}=-\sum_{j=1}^{N}\left[Z\left(t_{j}\right)\left(\frac{t_{j}}{\eta}\right)^{\beta-1} \ln \left(\frac{t_{j}}{\eta}\right) \exp \left(\gamma Z\left(t_{j}\right)\right)\right] \\
& \frac{\partial^{2} \ln L}{\partial \gamma \partial \eta}=\sum_{j=1}^{N}\left[\frac{\beta Z\left(t_{j}\right)}{\eta}\left(\frac{t_{j}}{\eta}\right)^{\beta} \exp \left(\gamma Z\left(t_{j}\right)\right)\right]
\end{aligned}
$$

Get a second-order derivative matrix, it can be represented as follows:

$$
H=\left(\begin{array}{lll}
\frac{\partial^{2} \ln L}{\partial \beta^{2}} & \frac{\partial^{2} \ln L}{\partial \beta \partial \eta} & \frac{\partial^{2} \ln L}{\partial \beta \partial \gamma} \\
\frac{\partial^{2} \ln L}{\partial \eta \partial \beta} & \frac{\partial^{2} \ln L}{\partial \eta^{2}} & \frac{\partial^{2} \ln L}{\partial \eta \partial \gamma} \\
\frac{\partial^{2} \ln L}{\partial \gamma \partial \beta} & \frac{\partial^{2} \ln L}{\partial \gamma \partial \eta} & \frac{\partial^{2} \ln L}{\partial \gamma^{2}}
\end{array}\right)
$$

Finally, newton-raphson method is used to construct the iterative formula, that is:

$$
\left(\begin{array}{l}
\beta \\
\eta \\
\gamma
\end{array}\right)_{K+1}=\left(\begin{array}{l}
\beta \\
\eta \\
\gamma
\end{array}\right)_{K}-H_{k}^{-1} g(k)
$$

$B_{0}, \eta_{0}$ and $\gamma_{0}$ is an initial value when $k=0$, determine a good initial value so that the value of three parameters can be solved according to the above iterative formula.

\section{Establishment Of Maintenance Decision Model}

Before determining the maintenance and control strategy of the key parts, it is necessary to make sure that what maintenance requirements need to be reached. In general, the key parts are required to have a maximum availability.

Availability can be defined as follows:

$$
K(t)=\frac{\text { availabletime }}{\text { availabletime }+ \text { unavailabd time }}
$$

The model based on WPHM can be established by combining the availability degree, so the availability will be updated as follows:

$$
K(t)=\frac{\int_{0}^{T} R(t, Z) d t}{\int_{0}^{T} R(t, Z) d t+R(T, Z) T_{p}+[1-R(T, Z)] T_{c}}
$$

Here definition a variable:

$$
\alpha=\frac{T_{d}}{T_{a}}=\frac{R(T, Z) T_{p}+[1-R(T, Z)] T_{c}}{\int_{0}^{T} R(t, Z) d t}
$$

where $T_{a}$ is represents an average running time of a component in a preventive maintenance cycle, $T_{c}$ is represents the average maintenance time afterwards, $T_{d}$ is represents the average downtime, $T_{p}$ is represents preventive maintenance time.

Then there is the following relationship:

$$
K(t)=\frac{1}{1+\alpha}
$$

In order to make the availability is maximum, $\alpha$ should be minimized. Specific solving process is to select a set of life history data, it is best to use full life of failure data to draw dot, along with features and each feature of the corresponding time substitute it into (24), and then by making the $\alpha$ - $t$ curve, fond the minimum $\alpha$ corresponds to at the time $T$, so this is the maximum points of availability, the maintenance threshold $h_{w}$ can be obtained by using the time point and the corresponding characteristic of the time points. Monitoring again for the same types of components, if the hazard is greater than $h_{w}$ when the obtained data substitute into (1), the components need to preventive maintenance. The relationship can be expressed as follows:

$$
h_{\mathrm{w}} \leq \frac{\beta}{\eta}\left[\frac{t}{\eta}\right]^{\beta-1} \exp \left(\gamma Z_{\mathrm{t}}\right)
$$

Taking logarithm on both side for (26) and will be updated it as follows: 


$$
\ln \frac{\eta h_{w}}{\beta}-(\beta-1) \ln t \leq \gamma \cdot Z_{\mathrm{t}}
$$

On the contrary, the device continues to run and monitoring, so the maintenance decision-making is based on the average maximum availability of the component.

\section{CASE STUdy}

Here have been collected 11 grinder spindle bearing life data from the MKS1620 series machine tool. After establish the WPHM, according to the maximum availability as the goal, carry out maintenance decision analysis, the life data shown in Table 1.

TABLE I. BEARING LIFE DATA

\begin{tabular}{cccccccccccc}
\hline Bearing Number & 1 & 2 & 3 & 4 & 5 & 6 & 7 & 8 & 9 & 10 & 11 \\
\hline Bearing Life/Day & 273 & 145 & 282 & 307 & 392 & 579 & 295 & 283 & 131 & 538 & 370 \\
\hline
\end{tabular}

In the experiment, a new bearing with the same type had been running the state monitoring, operation condition information of the key parts is obtained by acceleration senores. Here is use kurtosis value as the characteristic quantity of operation condition, which is closely related with the recession process of the bearing. While the kurtosis formula can be expressed as follows:

$$
Z=\frac{1}{N} \sum_{i=1}^{N}\left(\frac{x_{i}-\overline{x_{i}}}{\sigma_{i}}\right)^{4}
$$

where $N$ is the sampling length, $x_{i}$ is the signal values, $\bar{x}_{i}$ is the mean of signals, while $\sigma_{i}$ is the standard deviation of signals.

The new bearing is monitored and data are collected in a certain time interval, the value of kurtosis data as shown in Table 2. In Nine data, there are two failure data, remaining seven is the data after truncated, which meaning the system in monitoring point did not happen failure phenomenon.

TABLE II. RUNNING STATE DATA OF NEW BEARING

\begin{tabular}{ccccccccccc}
\hline Serial Number & 1 & 2 & 3 & 4 & 5 & 6 & 7 & 8 \\
\hline Monitoring Time/Day & 25 & 50 & 85 & 125 & 165 & 205 & 240 & 285 \\
\hline Value of Kurtosis & 2.306 & 2.871 & 3.025 & 4.736 & 6.218 & 9.439 & 12.650 & 14.024 & 17.489 \\
\hline
\end{tabular}

According to the Newton-Raphson method introduced in $\mathrm{B}$, combined 11 life data with state data of the new bearing, obtained the estimate value of three parameters $\beta=1.857$, $\eta=357.943$ and $\gamma=0.2831$. Therefore, the proportional hazards model of the main shaft bearing is obtained, which can be presented as follows:

$$
h(t, Z)=\frac{1.857}{357.943}\left[\frac{t}{357.943}\right]^{0.857} \exp \left(0.2831 Z_{\mathrm{t}}\right)
$$

If $T_{\delta} / T_{p}=10$, here can make the maintenance time is $10 \mathrm{~d}$, so the preventive maintenance time is $1 \mathrm{~d}$, and then plot $\alpha$ - $t$ curve, the curve obtained from the scanning point as shown in Figure 1.

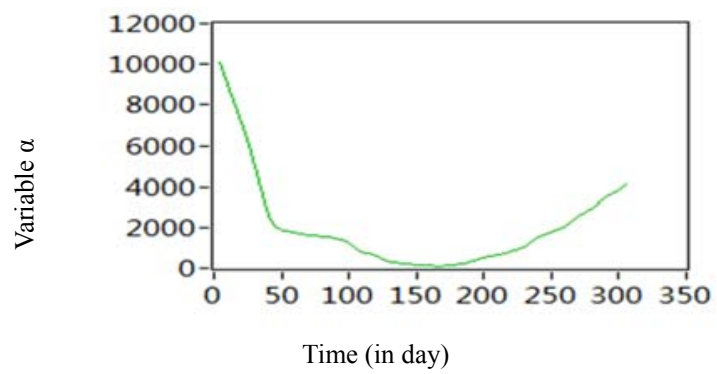

FIGURE I. $\alpha-t$ CURVE
It can be obtained from Figure 1 , when $T=165, \alpha$ is smallest, at this time, preventive maintenance can make the system to achieve the maximum availability, and then the failure threshold are obtained, the failure threshold can be calculated as follows:

$$
\begin{aligned}
& h_{w}=\frac{1.857}{357.943}\left[\frac{165}{357.943}\right]^{0.857} \\
& \exp (0.2831 \times 6.218)=0.028093111
\end{aligned}
$$

There is no doubt that the decision making process is too troublesome, in order to make the decision-making process more practical, make the system running time $t$ as horizontal coordinate, while the value of $\ln \left(\eta h_{w} / \beta\right)-(\beta-1) \ln t$ as vertical coordinate, drew Figure 2. 


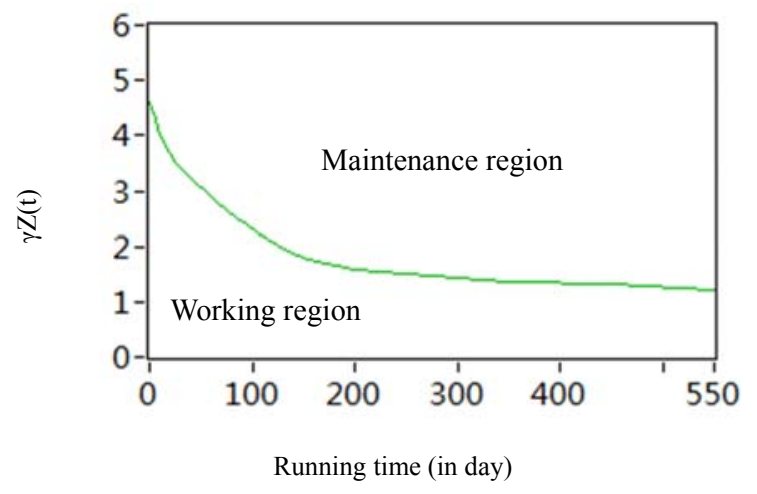

FIGURE II. MAINTENANCE DECISION DIAGRAM OF BEARING

Substitute the monitoring state data into right-hand of the (27), we can get some points with time, if the point that falls into the below region of curve, called the working region, at this time, the system continues to run and state monitoring. If the point that falls into the upper region of curve, called the maintenance region, at this time, the components need to preventive maintenance.

\section{CONCLUSIONS}

The on-line monitoring and maintenance decision model is obtained. This model can not only record the reliability and hazard of the components in real time, but also can maintain the components more reasonable, it can help enterprises to reduce maintenance costs, in line with the practical engineering significance.

A $\mathrm{W}$ based on condition information is established, the key problem is how to estimate the three parameters of the model. Here, the maximum likelihood estimation was used to estimate the parameters, which have a good advantage when the sample is incomplete.

In this paper, we used MKS1620 series grinder spindle bearings from a machine tool factory as an example. The results show that this maintenance decision model can improve the availability of the grinder spindle bearings greatly, and make full uses of the effective life for machine tools.

\section{ACKNOWLEDGMENT}

The authors declare that there is no conflict of interest. This work is supported by the National Science and Technology major projects of China (Grant No.2012ZX04010021-001-004) and Science and technology planning project of Beijing Municipal Education Commission (KZ201410005026, KZ201410005010). The authors are grateful to other participants of the projects for their cooperation.

\section{REFERENCES}

[1] X. C. Zhang, C. R. Li, X. X. Guo, "Reliability function of electronic equipments suffering a single environmental stress based on Poisson Process," IEEE Transl. J. Industrial Engineering and Engineering Management (IE\&EM), pp. 1143-1146, September 2011.

[2] S. M. Govind, O. A. Kobby, and D. H. Alan, "Transformation of the bathtub failure rate data in reliability for using Weibull-model analysis," J. Statistical Methodology, vol. 6, pp. 622-633, July 2009.

[3] B. A. Jaouher, C-M. Brigitte, S, Lotfi. M Simon, and F. Farhat,
"Accurate bearing remaining useful life prediction based on Weibull distribution and artificial neural network," J. Mechanical Systems and Signal Processing, vol. 56, pp.150-172, November 2015.

[4] C.C. Lin and H.Y. Tseng. "A neural network application for reliability modeling and condition-based predictive maintenance," J. Adv. Manuf. Technol, vol. 25, pp.174-179, January 2005.

[5] B. Lu, X. J. Zhou, and L. M. Y. Ting, "Joint modeling of preventive maintenance and quality improvement for deteriorating single-machine manufacturing systems," J. Computers \& Industrial Engineering, vol. 91, pp.188-196, January 2016.

[6] P. J. Vlok, J. L. Coetzee, D. Banjevic, A. K. S. Jardine and V. Makis "Optimal component replacement decisions using vibration monitoring and the proportional-hazards model," J. Journal of Operational Research Society, vol. 53, pp.193-202, February 2002. 